\title{
LOS ESPACIOS URBANOS RECREATIVOS \\ COMO HERRAMIENTA DE PRODUCTIVIDAD
}

\section{Resumen}

La temática que orienta el presente artículo es la identificación de las competencias urbanas en espacios recreativos donde se desarrollan actividades sociales y productivas, independientemente de su uso cotidiano.

Las características de la sociedad actual que vive en nuestro territorio urbano hace que el disfrute de los espacios públicos recreativos promueva cambios notables en el mercado laboral y en su fuerza productiva, en la globalización de la economía, el acelerado proceso de desarrollo tecnológico, el cada vez más ágil acceso a la información, además del contraste entre la búsqueda de un desarrollo económico y social sostenible, con el adecuado manejo del entorno y medio ambiente.

Los espacios recreativos denominados parques urbanos son un pretexto de análisis y clasificación por parte de las entidades competentes, e involucran a los actores y el entorno urbano de los parques activos, pasivos e interactivos sobre los cuales se planifican, implementan y desarrollan proyectos sostenibles productivos enmarcados en la política urbana ley 388 de $1997^{1}$ y demás decretos reglamentarios.

\section{Palabras clave}

Planificación, proyectos productivos, espacio público, competencias urbanas, productividad, ciudad y emprendimiento, competitividad, creatividad, regeneración urbana.
David Ricardo Ocampo Eljaiek*

\section{INTRODUCCIÓN}

\section{$\mathbf{A}$}

lo largo de las últimas décadas, las ciudades colombianas, y en especial Bogotá, han sido escenarios privilegiados de cambios físicos, socio - económicos, ambientales y políticos de gran alcance. Los desafíos planteados por las nuevas condiciones de la economía globalizada han tenido consecuencias importantes en la formulación y gestión de la política urbana y han forzado una redefinición de las prioridades y de los objetivos de la intervención pública en la ciudad.

La búsqueda de la competitividad y el aprovechamiento de los espacios urbanos se transforman en el principal argumento de la "nueva política urbana de acuerdo con la Ley 388 de 1997", y ésta se convierte en un instrumento clave de la gestión local de la economía global.

\footnotetext{
* Arquitecto, Magíster en Gestión Urbana, consultor de entidades públicas y privadas, profesor investigador Facultad de Humanidades y Ciencias Sociales, Universidad EAN, Profesor Universidad Piloto de Colombia, Facultad de Arquitectura, profesor Facultad de Arquitectura Pontificia Universidad Javeriana.

1 Ley de ordenamiento territorial, creada para unificar la visión del territorio.
} 
El desarrollo urbano que experimenta actualmente el territorio desde que se puso en marcha la Constitución Política Nacional de 1991 y se implementó el Ordenamiento Territorial bajo la Ley 388 de 1997 P.O.T, motiva que en los espacios urbanos se manejen dinámicas e implementen alternativas que involucren necesidades de disfrute y también de desarrollo territorial. Estos espacios no son productos del azar; son la respuesta física ante las necesidades, relaciones e interacciones sociales, económicas y ambientales, entre tantos atributos que contiene la ciudad. Por tanto, los parques urbanos activos, pasivos e interactivos son una necesidad, producto y resultado del desarrollo urbano, que permiten la planificación, implementación y desarrollo de proyectos formales e informales.

Este artículo analiza, en primer lugar, la transformación urbana motivada por la Ley de Ordenamiento Territorial 388 de $1997^{2}$, y su resultado, la competitividad urbana implementada en los espacios públicos recreativos, como referencia básica de emprendimiento.

Desde el punto de vista socioeconómico, la importancia estratégica de la ciudad y de los procesos urbanos de consolidación se vincula al reconocimiento de la competitividad global en el contexto territorial y se constituye en un componente fundamental de la capacidad de emprendimiento de las empresas, públicas, privadas y comunitarias. (Porter 1990; Storper, 1997)3. Esta concepción se enlaza con la idea

2 Ley 388 de 1997, denominada Ley de Ordenamiento Territorial, impulsa la organización lógica del territorio y se convierte en la alternativa de sostenibilidad de cualquier operación urbana en Colombia.

3 www.ucm.es/info/ec/jec10/ponencias/812Rodriguezetal.pdf

\section{Abstract}

The theme of this article shows the identification of urban competences in recreational sites where social and productive activities are carried out, in spite of their daily use.

The main features of our current society that occupies the urban spaces make the use of public recreational spaces promote significant changes in the work market and its productive forces, in the globalization of economy, the accelerated process of technological development, the fast and easy access to information, besides contrasting sustainable economic and social development with environmental management.

The urban recreational spaces known as urban parks are a real pretext to make an appropriate analysis and classification by the corresponding authorities, being passive and interactive, on which sustainable productive projects are designed, implemented and developed, according with the 388 regulation of 1997 and other legal agreements.

In the continuous search for improving and identifying territorial abilities and competences, which stimulates a permanent growth of urban society, we have to take into account the past, beliefs, methodological values, theoretical suppositions and processes of urban development to turn them into public recreational sites. This also requires a serious study to project all these experiences, conclusions and information in the design of entrepreneurial projects and urban productivity that really benefit future generations and the development of urban spaces itself.

\section{KEY WORDS}

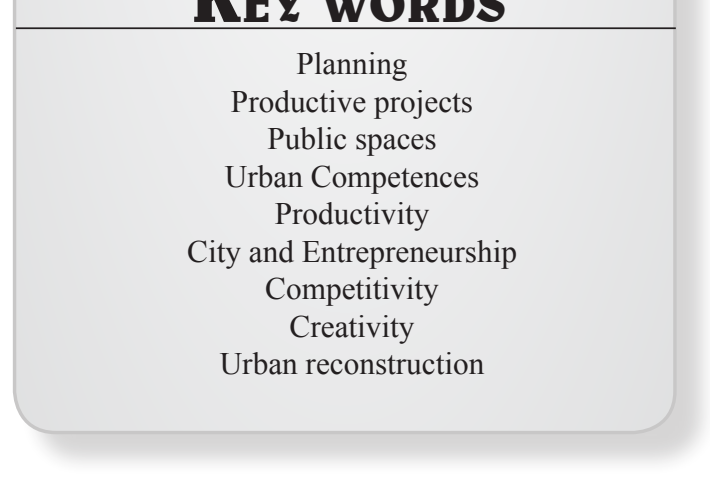


de que la productividad territorial estará sujeta a la economía local, que es de importancia como centro de gestión e implementación global (Borja y Castells 1997).Esta dinámica se plasma en tres ámbitos:

- Capacidad y rendimiento territorial.

- Competitividad económica formal e informal.

- Integración socio-cultural e integración de la política urbana.

En relación con la capacidad y rendimiento territorial el entorno urbano se revela como un elemento decisivo en la generación de ventajas en los espacios públicos creados, restaurados en el territorio luego de la puesta en vigencia de la Ley 388 de 1997, POT, como son los parques urbanos activos, pasivos, interactivos, públicos y privados $^{4}$. La ventaja competitiva de estos espacios se deriva no tanto de la eficiencia en el uso de factores productivos, sino de contar con técnicas del producto urbano (parques urbanos) y de los procesos de planeación.

Desde esta perspectiva, la innovación y disfrute de los espacios urbanos públicos se concibe como un proceso interactivo de aprendizaje en el que participa un conjunto de diversos agentes o actores: públicos, privados y comunitarios que interactúan por medio de una variedad de mecanismos y rutinas institucionales y de convenciones sociales que son específicas a cada entorno institucional (Lundvall, 1992), lo que significa que los procesos de aprendizaje son esencialmente endógenos y no pueden ser entendidos independientemente de ese contexto institucional (Morgan, 1997).
Esta adaptación de los espacios públicos urbanos" ya no son solamente una obligación de las políticas urbanas enmarcadas en la Constitución Política del 91 y ejecutadas por la Ley de Ordenamiento Territorial 388 de 1997 y sus respectivas normas y decretos de referencia, sino que el concepto admite espacios y actividades mucho más efectivas que posicionan a un territorio como un lugar privilegiado y permiten generar dinámicas económicas en entornos inmediatos, pues lo que caracteriza estos espacios recreativos ya no es su forma o su función sino su objetivo de construir un "lugar". En este sentido, algunos autores sugieren que en la nueva economía del conocimiento, donde la base económica de las ciudades son los servicios, el principal factor de competitividad y dinamismo de las economías urbanas y regionales es la presencia de actividad creativa. (Florida, 2000; Landry, 2000; Gertler, 2004), Bradford (2002) y otros (Gertler, 2004; Landry, 2000).

En el sentido funcional, se ha producido de forma análoga un desplazamiento de los contenidos y funciones, y se han mezclado diferentes posibilidades de recursos de esparcimiento y recreación. Los elementos naturales se han establecido como parte del paisaje, y en el ámbito social, la participación de los grupos heterogéneos de habitantes que participan en el territorio motiva las dinámicas económicas, formales e informales que existen en estos espacios urbanos reconocidos en el territorio.

Estas dinámicas están estrechamente vinculadas al uso del suelo ${ }^{6}$, y son representaciones frente a los poderes económicos, sociales y ambientales

\footnotetext{
4 Definiciones con base en la Ley 388 /97 POT.3 www.ucm.es/info/ec/jec10/ponencias/812Rodriguezetal.pdf.

5 Un ejemplo es el caso del Parque del Café, en Armenia, que involucra todo un paquete turístico, apoyado en el concepto de turismo para todo tipo de usuario, con base en una estructura cultural muy fuerte.

6 Usos del suelo, clasificación de las actividades económicas, sociales según el ordenamiento colombiano, Ley 388 de 1997.
} 
de la región y otros espacios de la estructura territorial colombiana; además, están vinculadas a las actividades de disfrute del espacio público.

En la actualidad, algunos de estos espacios públicos no se utilizan en absoluto para el fin para el que fueron construidos y diseñados; otros se han fortalecido al mezclar actividades que no tienen precisamente una relación directa con el espacio, sino que complementan sus actividades originarias y le dan un carácter recreativo, turístico y económico mucho más fuerte e importante: su función es exclusivamente la de crear espacios de reunión y recreación en las zonas urbanas no tenidas en cuenta por las entidades de emprendimiento, pero que potencialmente brindan muchas posibilidades.

El segundo ámbito se refiere a los factores competitivos, en los sectores formal e informal, que motivan a definir una infraestructura que incluye las redes sociales y los espacios que facilitan la interacción entre personas. Estos factores se consideran clave para atraer y aprovechar el capital humano que crea la infraestructura existente en el territorio urbano y la motivación al emprendimiento, que impulsa la innovación.

En los nuevos procesos productivos colombianos ${ }^{7}$, una parte importante de la creación de valor en muchos sectores se basa en activos intangibles, por lo que los factores locales decisivos en la nueva economía tienen que ver sobre todo con atributos y características de los lugares que los hacen especialmente atractivos para una clase creativa depositaria de talento. La creatividad y el talento depositado en una mano de obra muy educada desplazan a los factores tradicionales del crecimiento territorial: materias primas, suelo, redes de transporte, infraestructuras, etc., como base del desarrollo urbano.

\section{LOS ESPACIOS RECREATIVOS- UNA EXPRESIÓN}

$\mathbf{L}_{\text {os }}$

cambios por los que atraviesa el territorio han generado apreciaciones orientadas al cumplimiento del mandato constitucional y, en su defecto, la aplicación normativa de referencia; esto hace que se reconozca la historia reciente de la norma y la clasificación bajo los decretos $^{8}$ reglamentarios para poder contextualizar los términos normativos y las premisas en las que se entenderán estos espacios urbanos recreativo ${ }^{9}$ y la transformación de usos del suelo urbano y la economía de un entorno que no estaba estructurado para un fin de apoyo a este equipamiento colectivo, transformando la estructura física del entorno y una transformación en el uso del suelo.

\footnotetext{
En los últimos años varios factores han contribuido a aumentar la productividad laboral. Entre ellos se debe destacar el avance en la seguridad y la recuperación de la confianza que han permitido aumentar la tasa de inversión a niveles superiores al 25\% del PIB. La actualización de capital y la adopción de mejores tecnologías y mejores prácticas han implicado un aumento importante de la productividad factorial. Pág. 13 Informe Nacional de competitividad 2007 calidad y regulación (Capítulo 3) Colombia y Estados Unidos.

8 De acuerdo con el POT (Título II Componente urbano, Subtítulo 3. Los Sistemas Generales, Capítulo 10. Sistema de Espacio Público Construido: Parques y Espacios Peatonales, Subcapítulo 1. Parques distritales), la definición, clasificación y responsabilidad institucional en materia de parques en Bogotá. Referencia e información tomados de Funlibre / 2002. Derechos Reservados.

9 Artículo 230. Clasificación. El Sistema de Parques Distritales se clasifica así: IDRD . Plan Distrital de recreación 2003-2008, El IDRD como líder de la gestión sectorial. Bogota D.C. Plan Distrital de Desarrollo, Pág. 13.
} 
"En 2032, Colombia será uno de los tres países más competitivos de América Latina y tendrá un elevado nivel de ingreso por persona equivalente al de un país de ingresos medios altos, gracias a una economía exportadora de bienes y servicios de alto valor agregado e innovación, con un ambiente de negocios que incentive la inversión local ${ }^{10}$ y extranjera, propicie la convergencia regional, mejore las oportunidades de empleo formal, eleve la calidad de vida y reduzca sustancialmente los niveles de pobreza".

En definitiva, los nuevos tiempos vienen marcados por la importancia de la creatividad en el uso de estos espacios públicos urbanos recreativos y de la innovación, como fuerzas motoras del desarrollo económico y territorial.

La ciudad competitiva, creativa y emprendedora es la materialización de los nuevos territorios de prosperidad. Sin embargo, se afirma que la competencia entre ciudades es cada vez más intensa y por ello es necesario poner en marcha iniciativas que mejoren la capacidad innovadora de los lugares atrayendo a los sectores sociales y al capital humano adecuado: la "clase creativa".

En esta nueva era de la creatividad en el uso de las competencias, la producción de nuevos espacios urbanos adaptados a las necesidades de la comunidad es fundamental para la prosperidad de las ciudades y de los espacios públicos recreativos urbanos.
Sin embargo, estos espacios no son tan sencillos de reproducir. Se necesitan modelos que permitan implementar y formular proyectos productivos administrados por la comunidad innovadora que incluyan, entre otros, una nueva perspectiva de la intervención urbana, nuevas formas de administración y gobernabilidad, nuevos tipos de incentivos y el suministro de recursos culturales diversos (Landry, 2000), su transformación o desarrollo, y actividades económicas, sociales, y ambientales. También es necesario identificar cómo estos espacios se adaptan a los procesos mismos de transformación territorial, identificando y clasificando las actividades económicas formales e informales, dentro de esta búsqueda por entender el comportamiento del territorio, especialmente en los espacios públicos.

Los gestores públicos, privados y comunitarios permiten la toma de decisiones en el espacio público y que se dé respuesta a la concertación y apropiación, éstas como base y fundamento del origen de la ciudad ${ }^{11}$.

En términos de los factores de emprendimiento, la correlación entre espacio público urbano y actividades sociales y económicas determina los posibles proyectos de emprendimiento y desarrollo territorial e identifica los diferentes espacios y temporalidades.

En muchas ocasiones los parques urbanos cuentan con los medios económicos (suficientes para la ejecución), físicos, tecnológicos y humanos para el cumplimiento de las metas establecidas, lo que significa que se tienen en cuenta todos los factores que influyen en el logro de los propósitos.

\footnotetext{
10 Consejo Privado de Competitividad, Visión 2032 y principales estrategias. P.16.

11 Entendida como cuerpo social compuesto de individuos heterogéneos.
} 


\subsection{Competitividad en los espacios recreativos urbanos}

Entender el territorio y sus atributos facilita las condiciones de vida de la población. Ciertamente, las condiciones de vida o de bienestar en el territorio dependerán de una gran cantidad de factores, como son la satisfacción de las necesidades básicas, tales como alimentación, vestido, salud y vivienda, entre otras.

Sin embargo, hay otro grupo de necesidades que surgen dentro del proceso mismo del desarrollo: la educación y la recreación, entre otras, las cuales se han convertido en instrumentos indispensables para poder convivir socialmente.

Los sectores en que se involucra institucionalmente la recreación, requiere una intervención consistente con las características específicas de ese campo, pero ello obviamente demanda una coherencia y unificación del concepto de recreación y entender que la producción económica frente a la competitividad se refleja en aumentos en las entradas económicas, mayores ingresos por habitante y en una mejor calidad de vida de la población.

La intervención en estos espacios permite desarrollar criterios que están sujetos a las diferentes competencias, entendidas estas como un conjunto de conocimientos, cualidades, capacidades y aptitudes que habilitan para la discusión, la consulta y la decisión de todo lo que corresponde a un oficio, suponiendo conocimientos teóricos fundamentados, acompañados de las cualidades y de la capacidad que permiten ejecutar las decisiones sugeridas (Ropé, Tanguy, 1997).

Otro aspecto importante es que las competencias traducen una capacidad de abstracción, de desarrollo del pensamiento sistémico (que pretende superar la comprensión parcial y fragmentada de los fenómenos), de creatividad, de curiosidad, de pensar, en múltiples alternativas para la solución de un problema.

De este modo, la noción de competencias necesita ser entendida como una posibilidad multidimensional que involucra facetas que van de lo individual a lo sociocultural, situacional (contextual-organizacional) y procesual (Manfred, 1998).

Por este motivo, una competencia no puede ser confundida con el "desempeño" de tareas, principalmente cuando consideramos los espacios recreativos.

Es fundamental hacer una transposición de las competencias presentes en el proceso y en las relaciones establecidas, a una propuesta de formación, un diálogo entre los conocimientos y la experiencia concreta de la acción profesional que considera los valores, las historias y los saberes generados en la propia actividad de trabajo.

El concepto necesita ser ampliado porque la dimensión técnica no puede ser el único parámetro válido; las competencias no involucran solamente una dimensión individual de carácter cognitivo relacionada con los procesos de adquisición y construcción de conocimientos producidos por los sujetos ante las demandas de las situaciones concretas de trabajo.

La competitividad urbana involucra otra dimensión, fruto de experiencias colectivas, ya que representa una construcción influenciada por parámetros sociales, culturales, éticos, pedagógicos, políticos e históricos económicos (Schwartz, 2001). 
La noción de competencias urbanas se refiere, por lo tanto, a contextos, espacios y tiempos socioculturales, políticos y económicos; a transformaciones técnicas y organizacionales; a los impactos ambientales, a los saberes formales e informales de los propios usuarios y posibles trabajadores de los espacios recreativos, a las contradicciones de la sociedad, a los lazos colectivos y de solidaridad; es significativo también considerar las influencias de la clase social, género, credo, etnia, edad y grupos generacionales, entre otras.

En consecuencia, los procesos de identificación, definición y construcción de competencias urbanas en los espacios recreativos no deben ser dirigidos solamente a las demandas estrictas del mundo laboral, ya que éstas son dinámicas y aptas para transformaciones constantes, y normalmente, atienden los intereses que rodean el territorio.

A continuación se presenta una clasificación de los espacios urbanos recreativos con base en los decretos reglamentarios.

\subsubsection{Parques urbanos activos}

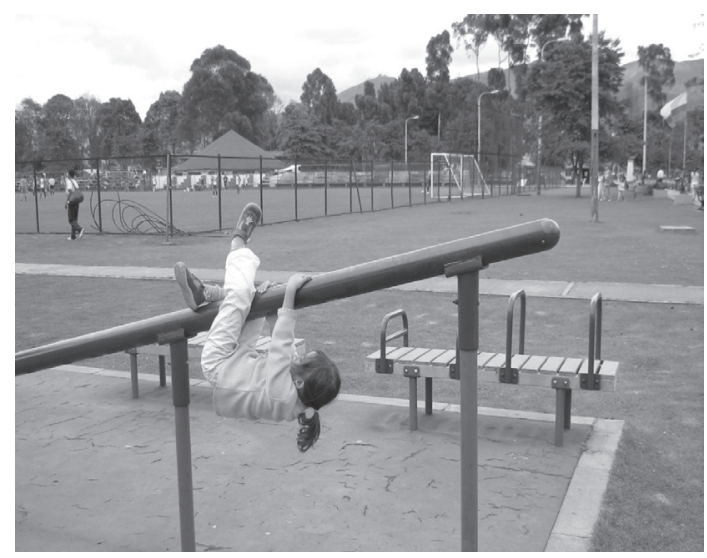

Parque El Salitre, Bogotá (Colombia). Foto del autor.
Son aquellos espacios físicos que pueden ser construidos, diseñados o reconstruidos para el desarrollo de actividades dirigidas al esparcimiento y al ejercicio de disciplinas lúdicas, artísticas o deportivas que tienen como fin promover la salud física y mental, y que requieren infraestructura destinada a concentraciones de público. Estos espacios sufren intervenciones paralelas a las actividades específicas del parque e invitan al sector informal ${ }^{12}$ a participar activamente. Algunas de estas actividades se encuentran registradas ante la Cámara de Comercio de Bogotá, y motivan al sector informal a generar proyectos de emprendimiento instintivo y poco planificado.

\subsubsection{Parques urbanos pasivos}

Parque El Virrey, Bogotá (Colombia). Foto del autor.

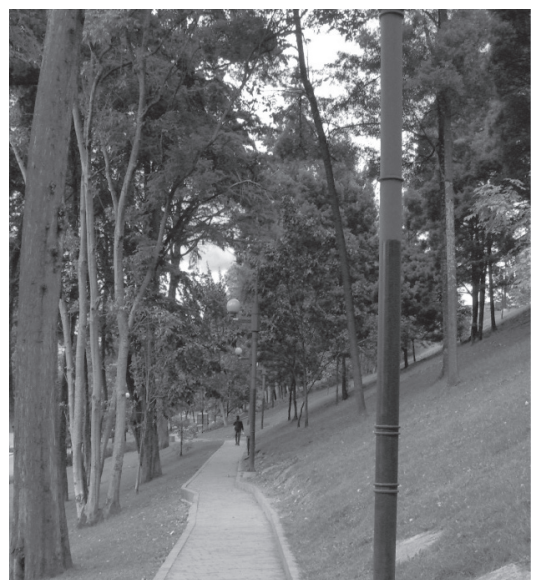

Son espacios físicos que se encuentran tanto en territorio urbano como rural, y están dirigidos a la realización de actividades contemplativas cuyo fin es el disfrute escénico y la salud física y mental; además, su impacto ambiental es muy bajo. Entre ellos se encuentran: senderos peatonales, miradores paisajísticos, observatorios de avifauna, y el mobiliario propio de las actividades contemplativas.

12 Días necesarios para empezar un negocio, 2006. 
Es muy difícil identificar espacios con tales características, puesto que generalmente en estos espacios también se llevan a cabo actividades que motivan el movimiento o el desarrollo psicomotor. Las diferencias radican en los usuarios que acostumbran visitar estos espacios; en este caso, estos espacios van dirigidos a usuarios que en promedio son adultos mayores con un auxiliar, o acompañados de niños y niñas de una edad inferior a 5 años o un máximo de 12 años.

\subsubsection{Parques urbanos interactivos}

Parque interactivo Play Land, Salitre Plaza, Bogotá (Colombia).

Foto del autor.

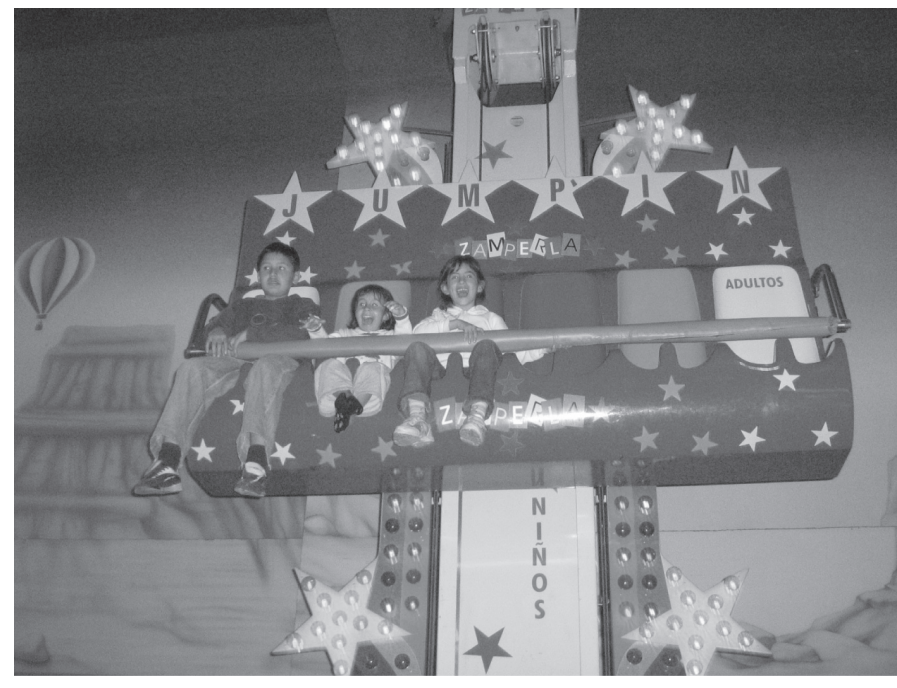

Estos espacios están concebidos para satisfacer las necesidades, deseos y expectativas de entretenimiento y esparcimiento de los diferentes grupos sociales, y buscan convertirse en su mejor opción, además de ser una herramienta eficaz de mercadotecnia integral para artistas, productores y prestadores de productos y servicios.

Un parque interactivo permite que el usuario disfrute de la más completa y variada diversión. Generalmente cuenta con atracciones mecánicas, juegos de destrezas, escenarios naturales, comparsas y personajes animados, entre otros.

Estos son sitios de aventura, diversión, alegría, emoción e interactividad donde el usuario aprende, a través de la experiencia, temas variados como la física, energía solar, ciencias, las plantas, informática, tecnología, comunicaciones, arte, el cuerpo humano y en general, el mundo que nos rodea. 


\subsection{Los espacios recreativos públicos urbanos y las relaciones espaciales en el entorno}

El hombre funda lugares incluso haciendo pequeñas modificaciones en las superficies y en los elementos que encuentra en su recorrido. Es a través del paso de otros hombres sobre el trazo 0 huella de un primero, o del paso reiterado de ese mismo hombre a lo largo del tiempo, que la huella se convierte en camino fundador; es así como surge el territorio.

Los parques urbanos y sus diferentes escalas en la ciudad representan múltiples dinámicas en el entorno, y sufren modificaciones que trascienden a la ciudad y su funcionamiento; de esta manera prefijan no sólo el disfrute, sino las maneras nuevas o ya consolidadas de potencializar el territorio con nuevas formas de pensamiento económico y todo lo que esa intervención conlleva.

Los entornos urbanos de los parques terminan estructurando las actividades de apoyo a la población, que se constituyen en un fenómeno normal debido al crecimiento de la ciudad y a la asignación del uso del suelo por parte de las Oficinas de Planeación, como respuesta a las condiciones de supervivencia que presenta la ciudad contemporánea.

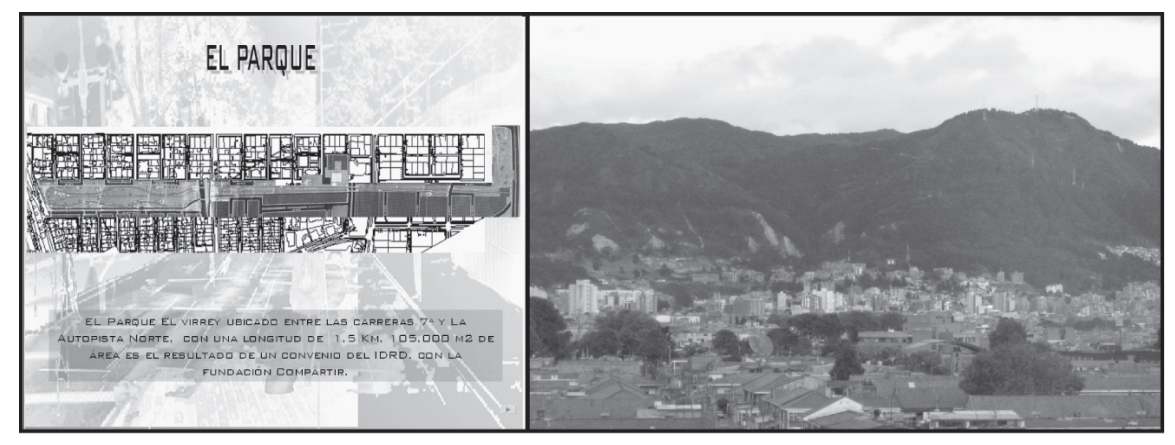

Entorno parques naturales, Cerros Orientales, Bogotá (Colombia).

Foto del autor.

El entorno urbano que está establecido junto a un parque urbano, activo, pasivo, e interactivo sufre múltiples evoluciones motivadas por las actividades que se realizan en estos espacios, y hacen que sea mucho más difícil la labor del planificador, en cuanto a la proyección de los cambios en el entorno.

Las actividades que se realizan impulsan y orientan la transformación del entorno. En Bogotá, se puede observar que el entorno de estos espacios está sujeto a factores como:
Población, usuario, temporalidad, ingresoeconómico, factores ambientales, actividades culturales, necesidades básicas y recuerdos entre otros; las dinámicas económicas dependerán de las actividades que se lleven a cabo en estos espacios, como por ejemplo las competencias deportivas a escala metropolitana e internacional, conciertos, días de campo, actividades educativas, de convivencia, interacción empresarial, barrial y familiar, desarrollo motriz, aeróbicos y todas las actividades recreativas activas o pasivas cuya influencia abarca todo el territorio urbano. 
Los cambios más típicos en el entorno de los parques activos, pasivos e interactivos están sujetos a las características de los usos del suelo, como por ejemplo, residencial, institucional, educativo, establecimientos de salud, de emer- gencia, espacio público, entre otros, y en este caso, los más típicos con base en la escala de usos de suelo establecidas por el Plan de Ordenamiento Territorial. Ver cuadro de características del entorno urbano del parque activo, pasivo e interactivo.

Esquema componentes del sistema urbano

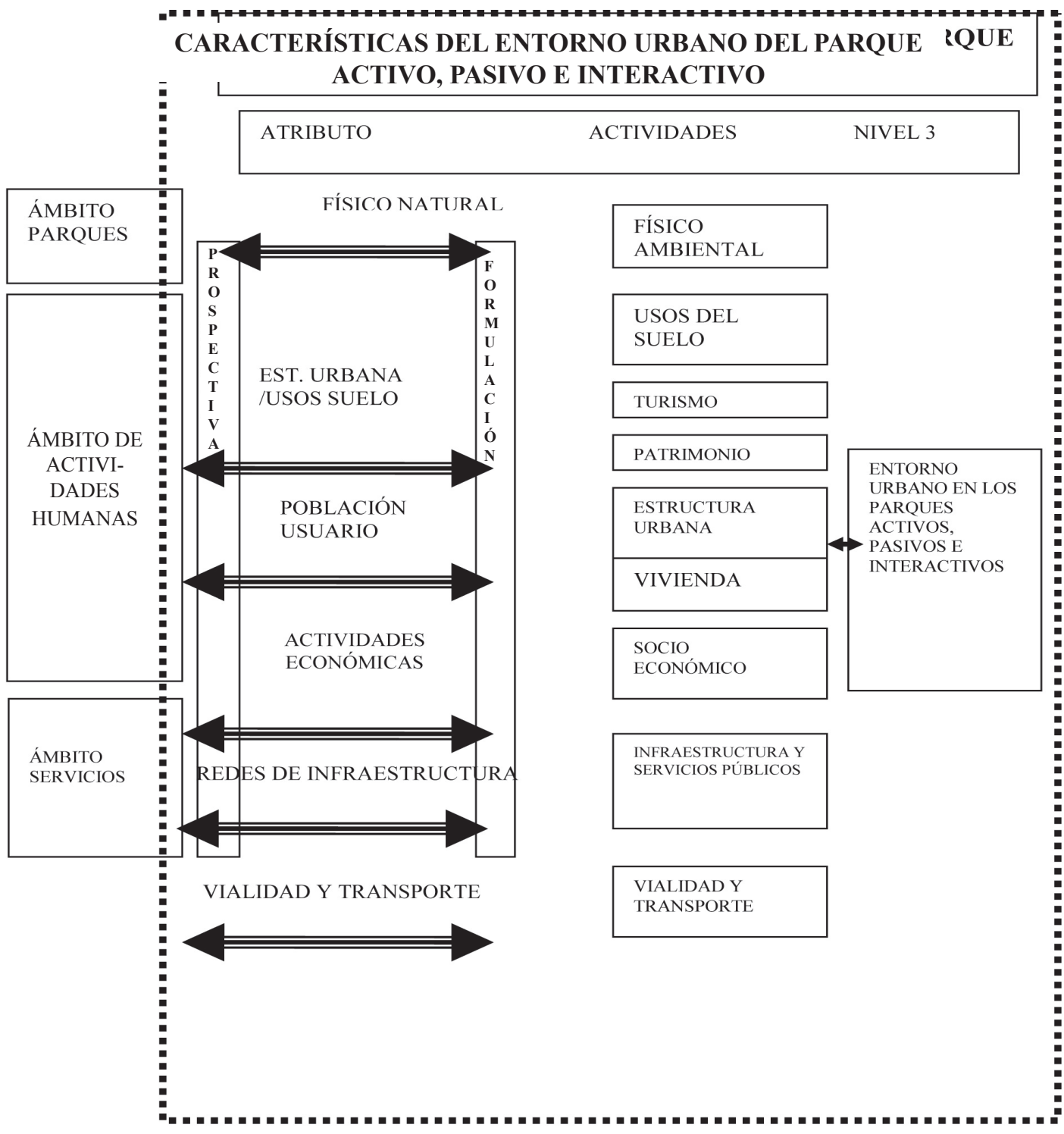

Estructura tomada e intervenida de los subsistemas de almacenamiento del PDUL de Altagracia como caso de estudio. Urbanismo Universidad Central de Venezuela y por el Instituto de investigaciones de la Facultad de Arquitectura Universidad del Zulia. 


\section{Enfoque y desarrollo. El disfrute urbano}

n este sentido, se consideró un enfoque basado en Mc Loughlin y Echeniche, presentado en los años 70 , en donde la ciudad es el resultado de un cúmulo de relaciones "espaciosactividades" y "comunicación-canales", y se la asocia a las concepciones actuales de desarrollo sustentable.

De esta consideración surge la reflexión sobre las operaciones urbanas en donde se establecen los componentes característicos del territorio, identificados a través de dos sistemas físicos:

- Por una parte, un sistema de espacios públicos adaptados que permite la evaluación de la capacidad de los espacios artificiales (edificaciones modificadoras del territorio) para contener diferentes usos del suelo (ya sean residenciales, industriales, recreativos).

- Un sistema de canales por medio del cual se confronta la intensidad de los flujos de servicio (ya sea de comunicación, de suministros o de evacuación) frente a la capacidad de los canales urbanos (de las redes y sus características).

Estos sistemas comprenden información susceptible de ser analizada y evaluada especialmente al comparar las distintas vocaciones de usos de suelo versus las capacidades límite de soporte para dichos usos. En este sentido, se conocen dos métodos:

\subsection{Método de superficies potenciales (Mcharg, 1969)}

Consiste en la generación de mapas síntesis de desarrollo potencial y conservación del medio ambiente, por medio de la superposición de capas de información especializada referente a las distintas variables del análisis urbano.

\subsection{Teoría de umbrales (Kozlowski, 1972)}

Permite calcular las capacidades límite de los servicios de infraestructura en la ciudad.

Una vez aplicados, es posible obtener la estruc-turación de la ciudad en sectores urbanos homogéneos, los cuales, una vez delimitados, se constituyen en la unidad especial básica de información para la planificación y la administración de cualquier espacio territorial.

Con base en las recomendaciones pertinentes a cada uno de ellos es que se establece una normativa de desarrollo apoyada en un plano de zonificación de usos del suelo, en un plan de vialidad y transporte, y en una serie de planos de ejecución por etapas de obras de infraestructura de servicios. 


\section{Condiciones urbanas}

\section{Ias ciudades se construyen en} torno a un espacio definido con características de abastecimiento, teniendo en cuenta los aspectos geográficos y socio-económicos. Su forma siempre está de acuerdo con las características de cada sociedad, pero las tendencias cambian con el tiempo y convierten la forma urbana en un arte temporal, un arte que cambia con las épocas, debido a que cada constructor que interviene en ellas modifica parte de su estructura, y ocasionalmente mantiene una relación con el entorno.

Esto explica por qué los países latinos asumimos las modalidades de nuestra historia. Las ciudades latinoamericanas se fundaron a partir del damero español, donde el centro básico o casco fundacional que estructura su desarrollo social y económico es la plaza o el parque, también llamado espacio público; es en estos espacios donde se llevó a cabo una porción importante de la socialización de nuestra cultura.
Este modelo, implementado desde el origen de la lógica de los cascos fundacionales, fue creado e implementado en Latinoamérica desde la óptica española mediante las Leyes de Indias, que a la larga fue la primera ley de ordenamiento territorial. Esta ley obviamente privilegiaba los intereses de los españoles, quienes privatizaban y distribuían la tierra sin detenerse a considerar la relación y las características naturales de su entorno inmediato y las características físicas del suelo.

En la Bogotá de hoy, luego de la implementación de diferentes formas de pensar, vivir y disfrutar la ciudad, traducidas en los diferentes desarrollos urbanos, se está llevando a cabo la implementación de un diseño normatizado con base en las directrices constitucionales, las leyes de referencia y las condiciones por las que tienen que pasar los diferentes espacios urbanos que surgen de modelos de implementación de todo el perímetro urbano.

\subsection{Escenario, escalas de intervención económica}

Los escenarios para la participación e intervención son los espacios en los cuales los miembros de la sociedad civil y la sociedad en general utilizan los parques urbanos en ejercicio de su derecho de disfrutar del territorio, y en cierta forma a motivar al cuerpo y a la mente a actividades diferentes de la rutina.

En estos espacios se llevan a cabo múltiples actividades de recreación, ocio, aprendizaje y comunicación ligadas a los patrones de desarrollo, con potencial económico formal e informal dentro de un solo espacio urbano.

Los usos del sector formal son aquellos sancionados por las entidades administrativas públicas; éstas cuentan con reglamentos creados para racionalizar el uso del suelo y brindar a la comunidad unos espacios distribuidos de manera que sea posible identificar el uso y 
el comportamiento de los ciudadanos en los diferentes espacios urbanos recreativos. Sin embargo, como señala Castells ${ }^{13}$, esta clase de planificación tiende a reforzar los intereses de los comerciantes posicionados dentro de los espacios productivos de los parques.

En nuestra ciudad, y en especial en los parques urbanos, tiende a presentarse la coexistencia de usos formales o informales, o no formalmente permitidos. En Colombia, el $60 \%$ del empleo está en el sector informal, cuya productividad es sustancialmente menor que la del sector formal.

Un trabajador formal produce en un día lo que un trabajador informal produce en 5,5 días de trabajo. No es posible aumentar la competitividad de la economía y disminuir la pobreza sin reducir la informalidad laboral y sin acelerar el tránsito hacia la formalidad empresarial ${ }^{14}$. Algunas de las actividades productivas comunes del sector informal en los espacios recreativos, parques y espacio público son las siguientes, con base en diagnósticos realizados en diferentes parques en Bogotá:

- Talleres ambulantes de mecánica de bicicletas.

- Puestos ambulantes de venta de refrescos y frutas, entre otros.

- Neveras ambulantes patrocinadas por entidades privadas, para venta de helados.

- Venta ambulante de algodón y dulces.

- Cuidadores de carros

- Venta de recreación activa (carros eléctricos, saltarines, carpas inflables, entre otros).

- Cometas, según temporada.

- Venta de comida típica, rápida, mazorca, pinchos, etc.
- Sombreros y cachuchas.

- Artesanías.

- Molinos de viento.

- Juegos para hacer bombas de jabón.

- Pinta caras para niños.

- Globos inflados con helio.

- Venta de minutos a celular.

- Venta de artículos deportivos.

- Publicidad de eventos.

- Robo de bicicletas y elementos deportivos, ropa y todo lo que esté al alcance.

El ejercicio de estas actividades dentro de estos espacios es una violación a la ley. Adicionalmente, el sector informal frena la expansión del sector formal, pues compite deslealmente al evadir el pago de impuestos, las cargas parafiscales, los aportes a la seguridad social, y en general, las regulaciones que recaen sobre el sector formal y los reglamentos que los pretende ubicar en sitios designados. Según la normatividad urbana, puede ser ilegal tener puestos ambulantes en zonas de comercios establecidos, como por ejemplo el entorno de los parques urbanos, sobre todo cuando los establecimientos consolidados legalmente están sujetos al pago de impuestos y arriendo entre otros.

Dado que la definición de informalidad empresarial se refiere a las actividades productivas legales que no cumplen todos los requisitos exigidos por el Estado, se puede decir que muchas unidades económicas desarrollan prácticas empresariales formales e informales simultáneamente.

Sin embargo, la informalidad se concentra principalmente en la microempresa y disminuye

\footnotetext{
${ }^{13}$ Castells, Manuel. La cuestión urbana, 1976. p 327, p. 475.

14 Informalidad y regulación, Pág. 44. Informe nacional de productividad, 2007.
} 
conforme va aumentando el tamaño de los establecimientos: en los establecimientos de un solo trabajador, únicamente el 17,8\% de los trabajadores está cubierto con algún tipo de protección laboral, mientras que en microempresas con 6 a 10 empleados dicha tasa es del $41 \%$. El porcentaje de protección se incrementa a medida que aumenta el tamaño de la empresa, pero aún en empresas con 50 ó más trabajadores, alrededor de un 10\% no tiene ningún tipo de protección laboral.

La informalidad empresarial se concentra en las microempresas, aunque también está presente en la mediana e incluso en la gran empresa.

El IDRD ha implementado una estrategia de sostenibilidad del sistema distrital de parques que incluye el tema de actividades y equipos de entretenimiento. Por ejemplo, para el caso específico de actividades con saltarines inflables, el procedimiento definido para la contratación de ofertas recreativas comprende la realización de un sondeo de mercado con el objetivo de identificar posibles asociaciones, personas jurídicas, consorcios o uniones temporales que desarrollen este tipo de actividad, así como la implementación de una prueba piloto y la contratación de una asesoría encargada de definir las actividades mecánicas, didácticas, interactivas y recreativas, entre otras, susceptibles de instalación y comercialización en parques administrados, así como las condiciones o requisitos que se deben cumplir en futuros procesos de participación a través de licitación pública ${ }^{15}$.

En general, los reglamentos sobre uso de espacios públicos, licencias de funcionamiento, etc., tienden a no ser respetados por el sector informal en la ciudad, lo que es evidente, aunque difícil de medir o cuantificar; sin embargo, el aprovechamiento de estosespacios representaaltísimos porcentajes del total de las actividades del uso del suelo ocupado en espacios recreativos, y se puede argumentar que si no fuera por el sistema complementario informal de uso del espacio, miles de personas estarían sin hogar o sin empleo, y una cantidad considerable de pequeñas y grandes empresas no estaría funcionando.

En este sentido, el sector informal brinda un complemento esencial y válido que suplementa los esfuerzos insuficientes de gobiernos que no tienen ni recursos ni voluntad política para suplir todas las necesidades de sus habitantes. Se trata de sistemas que seguirán frustrando la planificación racional del uso del suelo y de otros aspectos de la vida en el territorio urbano, mientras no se desarrollen métodos para captarlos oficialmente como herramientas legítimas.

Los elementos reconocidos legalmente establecen la participación de la comunidad en el disfrute económico que ofrecen los espacios públicos, y permiten equilibrar económicamente el ingreso de los habitantes de un sector en términos de economías derivadas de la recreación, y en este caso, de los parques como espacios urbanos productivos. Esta permanente y sospechosa tendencia al comercio informal debería plantearnos necesariamente una forma diferente de acceder al estudio de los hechos sociales, políticos y culturales; no sólo por la inevitable condición de parcialidad de la informalidad, ni por el peso estadístico y de proporción de la informalidad, sino por el reconocimiento de formas diversas de coexistencia individual y grupal.

\footnotetext{
${ }^{15}$ Mayo 13 de 2004, preguntas frecuentes IDRD.
} 


\subsection{Coexistencia y tolerancia}

Colombia es uno de los países con mayor nivel de informalidad económica en América Latina, No se trata de legalizar, normalizar o incorporar los amplios sectores informales que habitan en los espacios públicos y que están por fuera de las condiciones preestablecidas por las normas y las políticas urbanas; se trata de recuperar una lógica de reconocimiento que permita la existencia de otras formas de ocupación y de manejo económico de estos espacios.

Participación de los trabajadores informales en el empleo total

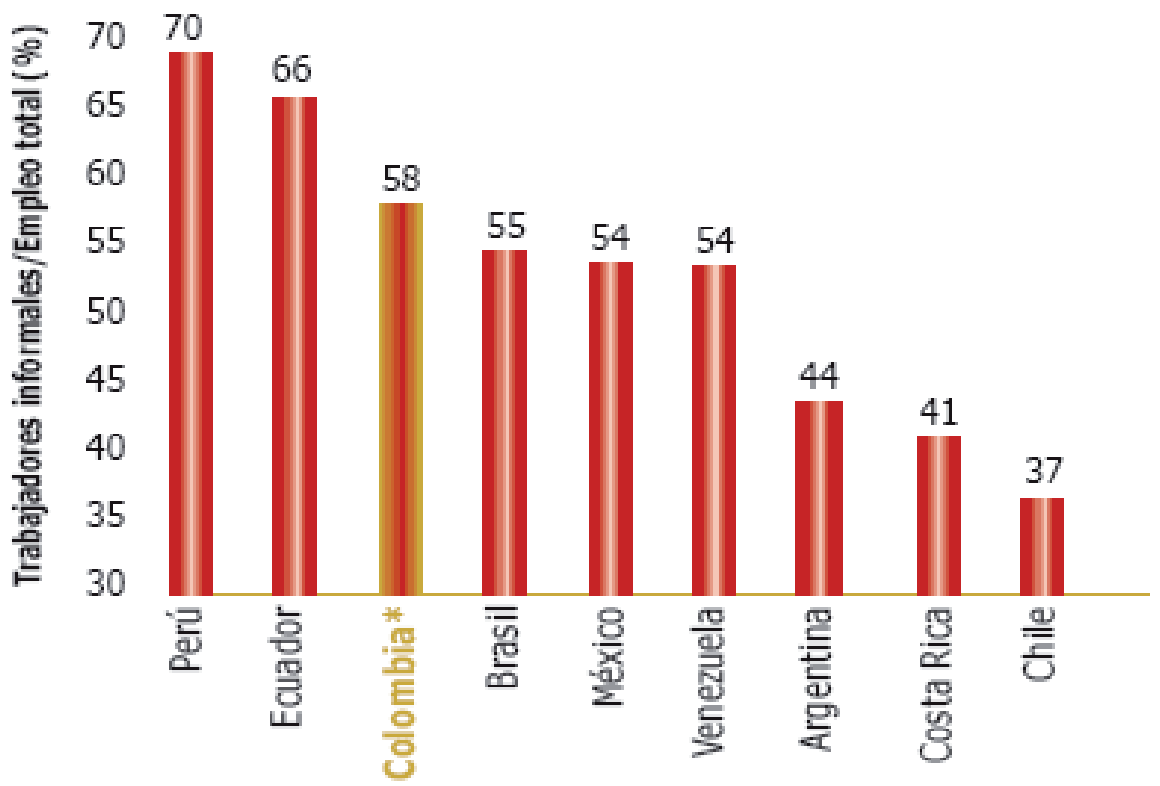

Fuente: informe nacional de competitividad 2007. 


\section{FACTORES DE EMPRENDIMIENTO ANÁLISIS CONCEPTUAL CON BASE EN EL ENTORNO}

I documento "Ciudades y Ciudadanía1995" planteaque "laglobalización impone una mayor competitividad de los sectores económicos y de sus territorios y ciudades (...) y que se requiere que los centros urbanos adapten sus bases económicas y condiciones de infraestructura, de tal manera que logren ser competitivas". Sin embargo, cada vez hay nuevas responsabilidades y una mayor escasez de recursos, especialmente en el área social, lo cual no le permite al territorio ser viable financieramente; se contraen nuevas deudas y se aumentan los impuestos, lo que afecta negativamente los renglones productivos. Por esta razón, se requiere plantear modelos de emprendimiento que consideren estas condiciones.

La prestación de un servicio con equidad implica viabilizar e involucrar a los integrantes de la sociedad sin limitaciones por sus condiciones, características y edad. Un sistema de oferta de programas y servicios recreativos equitativo y justo socialmente no puede dejar al azar el diseño de sus programas. La cultura, la perspectiva de género, los niveles de madurez y desarrollo por ciclo vital, las diferentes esferas del desarrollo humano, son condiciones que necesariamente se deben tener en cuenta.

Se estima conveniente que en el quehacer de la recreación y para poder determinar proyectos productivos sostenibles, se adelante en primera instancia una segmentación poblacional, tomando como referencia las fases del ciclo de vida.
En consecuencia, se contemplan cinco segmentos:

- Niños.

- Jóvenes.

- Adultos.

- Adultos mayores.

- Discapacitados.

En segunda instancia, en estos segmentos es posible hacer una nueva división, basada en los grupos que demandan una atención especial en virtud de sus particularidades y necesidades en equidad de género, condición y etnia.

Se debe enfatizar que aunque estas categorías generales pueden servir como referente para la formulación de planes, programas y proyectos de emprendimiento, deben ser suficientemente flexibles en su aplicación de tal manera que no se masifiquen las propuestas y se puedan ajustar a las necesidades y expectativas específicas de participantes particulares.

En países y ciudades con nuestras condiciones, la inversión social se orienta a la población en condiciones de vulnerabilidad, en muchos casos, principalmente económica, lo que ocasiona que se "descuiden" segmentos que si bien han logrado condiciones de vida o un nivel satisfactorio en la realización de sus necesidades básicas, tienen carencias en la realización de necesidades más complejas, o están expuestas a ofertas de ocio y recreación que no promueven el desarrollo humano. 
La gestión de proyectos sociales incluye la aplicación de normas desde la planeación, la ejecución y el seguimiento de proyectos. El desarrollo y aplicación de normativas hace parte de los instrumentos de gestión, y son básicas para la ejecución de mecanismos de concertación, control y gestión social. En este artículo de investigación se dejan las puertas abiertas para que el lector se dé la oportunidad de pensar un poco más en las condiciones de transformación y en las nuevas oportunidades con que cuenta nuestro territorio, e invita a participar en la reflexión sobre los parques urbanos como espacios productivos, y a aportar ideas acerca de cómo podemos posicionar nuestras unidades territoriales tan importantes para nuestra identidad cultural.

\section{Conclusiones y Recomendaciones}

\section{sta investigación es un buen} pretexto para orientar la participación de todos los gestores públicos, privados y comunitarios, e involucrarlos o hacerlos partícipes en los grandes cambios y/o posibilidades de emprendimiento que se presentan en el territorio, y para que utilicen los mecanismos de gestión en las condiciones que requiere la formación científica y tecnológica, de manera que sea posible dar una dirección al desarrollo de proyectos productivos que brinden soluciones significativas a las necesidades del futuro de la ciudad y de quienes la habitan.

El producto denominado parque tiene la fortuna de aparecer en el territorio gracias a los procesos sociales, culturales, económicos y ambientales, y la planificación del desarrollo de los mismos no puede fundarse en la aparente pretensión de construir palmo a palmo el futuro de cada barrio o localidad, ni mucho menos de una gran ciudad, tal como se creía en los viejos enfoques ; por el contrario, la formulación y ejecución de un Plan de Ordenamiento debe ser un procedimiento mediante el cual quienes tienen el poder de tomar las decisiones clave en cada jurisdicción (y esto incluye a los inversionistas, las autoridades y a los dirigentes políticos) puedan entrever qué tipo de implicaciones ambientales, urbanas, políticas - institucionales y socio - económicas, pueden derivarse para el futuro previsible, de las elecciones que están realizando en cada momento concreto, para que estos agentes puedan racionalizar las decisiones que toman, en función de las consecuencia previsibles.

La urbe pretende brindar el marco físico para una colectividad urbana. Sin embargo, el tema del espacio público recreativo se ha desconocido o evadido, a pesar de ser éste generalmente un espacio resultante de las necesidades urbanas. Por esta razón, siempre se ha asociado los espacios recreativos con lo gratuito, y se ha cometido el error de relegarlos. No obstante, el sector informal los ha aprovechado, y como resultado se generan lugares $\sin$ infraestructura para estas actividades.

La optimización de la política urbana parte de la definición de un modelo de ciudad que va a ser integrado a las políticas e inversiones de las entidades competentes en los centros urbanos, quienes deben responder a la problemática descrita. De esta forma, se busca lograr ciudades productivas, en la medida en que esta configuración de los espacios públicos recreativos estimule la concentración de actividades económicas participativas, disminuyendo los desplazamientos entre las áreas residenciales, los servicios urbanos y el empleo. 


\section{Bibliografía}

Sallenave J.(1985): Gerencia y planeación estratégica.Bogotá.

Rojas E.(1998): La ciudad siglo XXI. Experiencias exitosas en gestión del desarrollo urbano en América Latina. IDB Bookstore.

Richards Ben. La ciudad siglo XXI. Reconstrucción de la urdimbre social.

Etchegaray A. La reconstrucción de la trama social.

Valenzuela A. (1999): Maestría en Gestión Urbana Alternativas de Gestión para proyectos de transporte urbano.

Plan Nacional de Desarrollo del Gobierno de Turno 1.998-2.002: CAMBIO para CONSTRUIR la PAZ" (Ley 508/99.

Constitución Política de Colombia. Editorial Leyer.

Página oficial del Congreso de la República y Ministerios, por medio del servidor altavista:

Ley 388 de 1993. Ordenamiento territorial.

Ley 152 de 1994. Ley Orgánica del Plan de Desarrollo.

ALLENDE, J. (2005) "Zorrotzaurre: urbanismo de maqueta", El País, 4 de enero.

www.bilbao.net/castella/bilbaonegocios/invertir/ciudad/oportunidades (2004) Plan Especial de Bilbao La Vieja, San

Bell, D. Y Binnie, J. (2004): "Authenticating queer space: citizenship, urbanism and governance" Urban Studies, vol.41.9, pp.1807-1820. West European Experience. Manchester: Manchester University Press.

Bilbano Metrópolil-30 (BM) (1999) Bilbao 2010. Reflexión Estratégica. Bring your dreams to Bilbao. Bilbao: Bilbao Metrópoli-30. (2000) Informe 2000: Una Década de Revitalización en el Bilbao Metropolitano. Bilbao: Bilbao Metrópoli-30.

Egidio, J.A. (2001): "La actividad de los lobbys privados en relación a la administración Pública. Un estudio de caso en Bilbao", Inguruak, núm. 30, p. 185-194. 
Esteban, M. (2000): Bilbao, Luces y sombras del titanio. El proceso de regeneración urbana del Bilbao Metropolitano. Bilbao: Servicio Editorial Universidad del País Vasco. vol. 9, p. 851-869. University Press.

Nieva, A. y NG, W. (2005): "Innovar, el reto de la ciudad". El Correo, 16 de octubre.

Porter, M. (1990): The Competitive Advantage of Nations. Londres: MacMillan.

Porter, M. (1998): "Clusters and the new economics of competition". Harvard Business Review, Nov-Dic, p. $77-90$.

Rodríguez, A. (2002): "Reinventar la ciudad: milagros y espejismos de la revitalización urbana en Bilbao", Lan Harremanak, núm. 6, p. 69-108. 


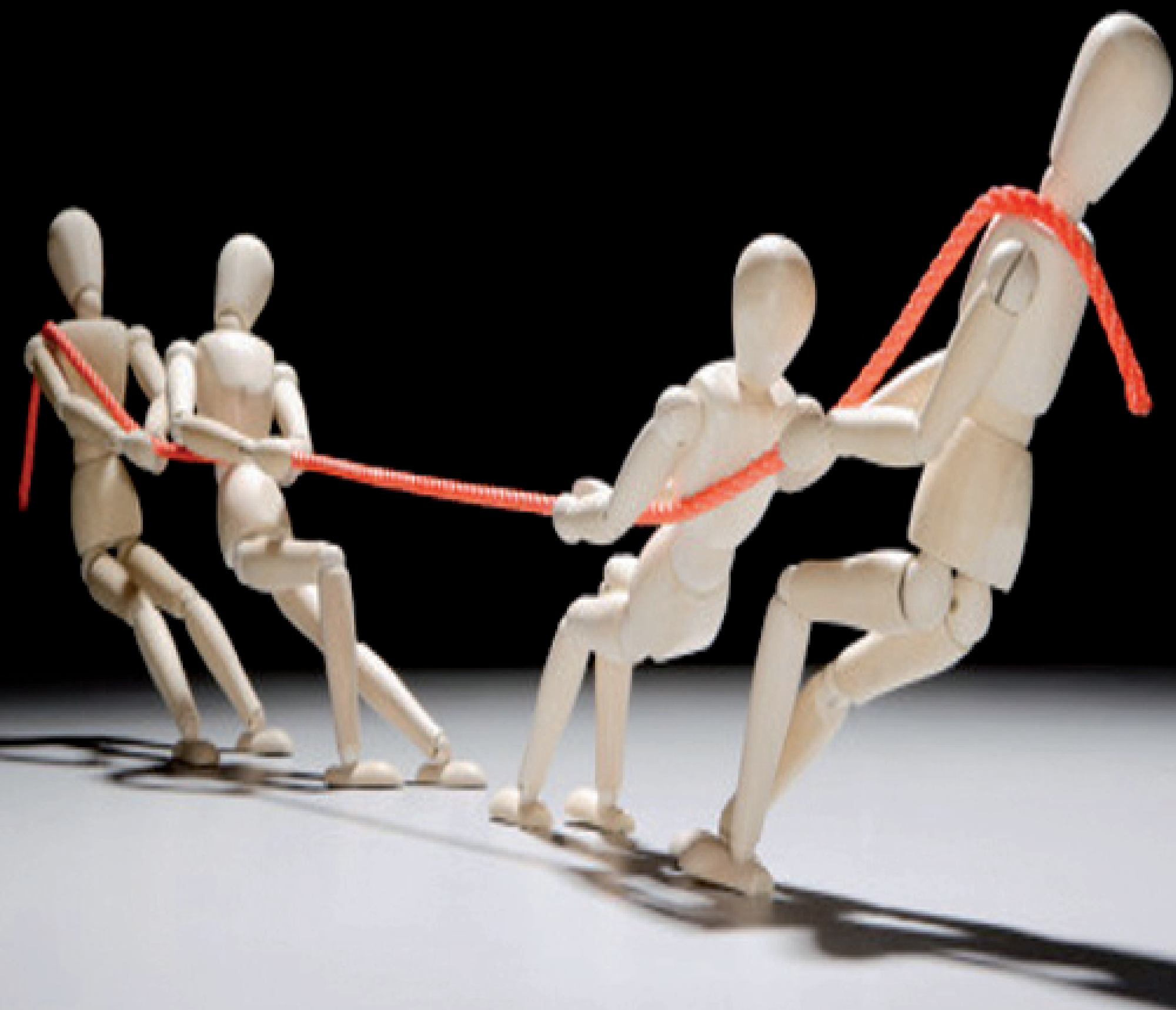

